\title{
Low Dose Radiotherapy to Thorax for COVID-19 Patients: A Potential Weapon to Put the Dagger in Devil's Heart
}

\author{
Aman Sharma, Daya Nand Sharma
}

Department of Radiation Oncology, National Cancer Institute-AIIMS, Jhajjar, Haryana, India.

\begin{abstract}
The novel coronavirus SARS-Cov-2/COVID-19 pandemic has emerged as a major healthcare burden to the entire world. At the moment there is no specific antiviral treatment recommended for COVID-19, and no vaccine is currently available. The clinical spectrum of SARS-CoV-2 infection ranges from asymptomatic infection, mild upper respiratory tract illness to severe pneumonia with respiratory failure, multi organ failure and death. The inflammatory response induced in pneumonia is complex and involves a variety of mechanisms to defend against pathogens and repair tissue. During inflammation, activation of inflammatory cells releases cytokines and this intense inflammatory reaction can further to life threatening condition know acute respiratory distress syndrome. In the past, low dose radiotherapy has successfully cured patients with unresolved viral pneumonia. It was associated with reduction of mortality in unresolved pneumonias. Radiotherapy at low doses exerts anti-inflammatory effects which have potential to reduce the cytokine storm in COVID pneumonia patients. We hereby briefly touch upon COVID-19 infection and potential of low dose radiotherapy to reverse unresolved pneumonia, prevent development of acute respiratory distress syndrome as well as multi-organ failure.
\end{abstract}

Keywords: COVID-19- coronavirus- pneumonia- low dose radiotherapy

\section{Introduction}

The last two decades have witnessed viral epidemics such as severe acute respiratory syndrome coronavirus (SARS-CoV) in 2002-03, H1N1 influenza in 2009, Middle East respiratory syndrome coronavirus (MERS-CoV) in 2012 [1]. Recently, novel coronavirus (SARS-Cov-2/ COVID-19) has caused a viral pandemic, that has now become major healthcare concern to the entire world. This virus is highly contagious, human to human transmission occurs through respiratory droplets. The clinical spectrum of SARS-CoV-2 infection include a wide range of symptoms. This can be asymptomatic infection, mild upper respiratory tract illness, and severe viral pneumonia with respiratory failure, multi organ failure and death [1]. Recently sepsis has been identified as the most frequently observed complication, followed by respiratory failure, ARDS, heart failure, and septic shock [2].

The pathogenic mechanism that produces COVID-19 pneumonia are complex. In viral infection, viruses trigger immune cells to synthesize pro-inflammatory cytokines and chemokines inciting the immune response [3]. This intense inflammatory reaction as a whole is often referred a 'cytokine storm'. The effect is extensive tissue damage. The protagonist of this storm is interleukin 6 (IL-6). IL-6 is produced by activated leukocytes and acts on a large number of cells and tissues. It is also implicated into the pathogenesis of the cytokine release syndrome that is an acute systemic inflammatory syndrome characterized by fever and multiple organ dysfunction [1].

At the moment there is no specific antiviral treatment recommended for COVID-19, and no vaccine is currently available. The treatment is symptomatic, and oxygen therapy represents the major treatment intervention for patients with severe infection. Mechanical ventilation may be necessary in cases of respiratory failure refractory to oxygen therapy, whereas hemodynamic support is essential for managing septic shock [1].

\footnotetext{
Corresponding Author:

Dr. Aman Sharma

Department of Radiation Oncology, National Cancer Institute-AIIMS, Jhajjar, Haryana, India.

Email: amans757@gmail.com
} 


\section{Radiation hormesis}

Low dose radiation therapy (LDRT) has been successfully used to treat viral pneumonia [4-6]. The positive effects of radiotherapy have been attributed the phenomena known as radiation hormesis. Radiation hormesis is defined as a hypothesis where low doses of radiation (just above natural background levels) is beneficial, stimulates activation of repair mechanisms that protect against disease, that are not activated in absence of ionizing radiation [7]. The beneficial effect of LDRT were first described by Olivieri et al when they demonstrated that the adaptive response induced by low-dose radiation, induced cellular resistance to genotoxic effects caused by subsequently high-dose radiation (HDR) [8].

\section{Properties of LDRT}

Although, experimental studies pointing the exact underlying cellular and molecular mechanisms in this subset of disease are still rare and fragmentary, LDRT has clinically exerted anti-inflammatory effect on several benign disease and chronic degenerative disorders [9]. The modulation by LDRT on immunological response have been explored in vitro and in vivo [10]. These include leukocyte/endothelial cell adhesion, adhesion molecule and cytokine/chemokine expression, apoptosis induction, and mononuclear/polymorphonuclear cell metabolism and activity [10]. These mechanisms display comparable dose dependences and dose-effect relationships and are observed when radiation dose is $<1 \mathrm{~Gy}$, maximum effect is seen in range of 0.3-0.7 Gy [10].

\section{LDRT in Pneumonia}

The inflammatory response induced in pneumonia is complex and involves a variety of mechanisms to defend against pathogens and repair tissue. During inflammation, numerous types of inflammatory cells are activated. Each releases cytokines and mediators to modify activities of other inflammatory cells [3]. This acute inflammation manifests as pneumonia or acute respiratory distress syndrome (ARDS). Because the lung is a vital organ for gas exchange, excessive inflammation can be life threatening.

In the pre-antibiotic era studies were conducted on animal models (guinea pigs, dogs cat and mouse) to assess the efficacy of LDRT for bacterial and viral pneumonias [4-11-12]. These studies supported the hypothesis that LDRT could reduce the effects of the pneumonia induced by bacteria or viruses and demonstrated that early initiation of LDRT was more efficacious in recovery of pneumonia [4].

In humans, LDRT successfully treated viral and bacterial pneumonia in the pre antibiotic era [4-6]. LDRT effectively cured pneumonia and relieved respiratory distress. Not only did it reverse pneumonia but also deceased mortality. There is significant reduction in mortality rate ( $30 \%$ vs $5-10 \%)$ in pneumonia patients with use of LDRT [4]. In a recently published review Calabrese et el reviewed 15 studies report that approximately 700 cases of bacterial, sulfanilamide non-responsive, interstitial, and atypical pneumonia were effectively treated by low doses radiotherapy. LDRT resulted in disease resolution, based on clinical symptoms, objective disease biomarkers, and mortality incidence. The study concluded that LDRT caused rapid reversal of clinical symptoms, facilitating disease resolution and that LDRT has capacity to suppress inflammatory responses which has widespread biomedical and therapeutic applications [4].

\section{LDRT in COVID pneumonia patients}

In the recent past LDRT has seldom been used to treat unresolved pneumonias and at the moment there is lack of robust evidence for suggest efficacy of LDRT against viral pneumonia. But, LDRT is effective and efficacious in treatment of viral pneumonia. Use of LDRT has resulted in significant reduction of morbidity and mortality for patients with viral pneumonias [4]. Never in the last century has mankind witnessed such a highly infectious viral pandemic with high mortality. Given the lack of any effective treatment and high mortality, LDRT seems a reasonable option to explore in moderately symptomatic (before the release of cytokine storm) COVID-19 patients with pneumonia. LDRT with its immune-modulatory properties has potential to resolve the pneumonia and reduce the cytokine storm which will prevent development of ARDS and reduce mortality.

\section{Logistics in delivery of LDRT}

There are ethical issues and logistics involved in to deliver radiotherapy in COVID-19 pneumonia patients. Treating symptomatic COVID-19 patients with pneumonias requires a dedicated radiotherapy facility as this poses risk of infection and contamination to the staff and radiation therapy unit. Disinfection of the radiation therapy machine and its room is possible but is technically demanding. Reducing risk of contamination during transport of COVID-19 patients from isolation to radiotherapy facility requires special prevention and disinfection protocols. There could also be apprehension regarding increased risk of second malignancy with use of LDRT among patients as well as the oncology and non-oncology fraternity. This is especially true if we assume that delivery of radiotherapy follows a linear no threshold (LNT) model. However, at low doses reduction of damage by adaptive protection equals to or outweighs radiogenic damage [13]. The LNT hypothesis for cancer risk appears to be scientifically unfounded and invalid in favor of a threshold or hormesis as observed within data from animal studies and human epidemiological observations on low-dose induced cancer [13]. Low doses radiotherapy is protective and is not associated with significant risk.

LDRT to the lungs appears to be a promising therapeutic option to be explored in this vicious viral pandemic that at the moment has no definite treatment. LDRT can rapidly reverse pneumonia and prevent cytokine storm (that leads to ARDS and multi-organ failure). Furthermore, it has potential to reduce burden on healthcare systems (oxygen dependence, need of intubation, reducing saturation of ICU \& ventilator support). 


\section{References}

1. Cascella M, Rajnik M, Cuomo A, et al. Features, Evaluation and Treatment Coronavirus (COVID-19); StatPearls Publishing: Treasure Island, FL, USA.. 2020;.

2. Zhou F, Yu T, Du R, Fan G, Liu Y, Liu Z, Xiang J, Wang Y, Song B, Gu X, Guan L, Wei Y, Li H, Wu X, Xu J, Tu S, Zhang Y, Chen H, Cao B. Clinical course and risk factors for mortality of adult inpatients with COVID-19 in Wuhan, China: a retrospective cohort study. The Lancet. 2020 03;395(10229):1054-1062. https://doi.org/10.1016/s01406736(20)30566-3

3. Moldoveanu B, Otmishi P, Jani P, et al. Inflammatory mechanisms in the lung. J Inflamm Res. 2009;2:111.

4. Calabrese E, Dhawan G. How radiotherapy was historically used to treat pneumonia: Could it be useful today? . Yale J Biol Med. 2013;86:555-70.

5. Oppenheimer A. Roentgen therapy of interstitial pneumonia. J Pediatr. 1943;41:404-14.

6. Dubin I, Baylin G, Gobble W, Jr. The effect of roentgen therapy on experimental virus pneumonia; on pneumonia produced in white mice by swine influenza virus. Am J Roentgenol Radium Ther 55. 1946;:478-81.

7. Liu S. Radiation hormesis. A new concept in radiological science. Chin. Med. J. (Engl.). 1999;102(10):750-5.

8. Olivieri G, Bodycote J, Wolff S. Adaptive response of human lymphocytes to low concentrations of radioactive thymidine. Science. 198402 10;223(4636):594-597. https:// doi.org/10.1126/science.6695170

9. Cuttler JM. Application of Low Doses of Ionizing Radiation in Medical Therapies. Dose-Response. $20200101 ; 18(1): 155932581989573$. https://doi. org/10.1177/1559325819895739

10. Rödel F, Frey B, Manda K, Hildebrandt G, Hehlgans S, Keilholz L, Seegenschmiedt MH, Gaipl US, Rödel C. Immunomodulatory Properties and Molecular Effects in Inflammatory Diseases of Low-Dose X-Irradiation. Frontiers in Oncology. 2012;2. https://doi.org/10.3389/ fonc. 2012.00120

11. Lieberman L, Hodes P, Leopold S. Roentgen therapy of experimental lobar pneumonia in dogs. American Journal of the Medical Sciences. 1941;291(1):92-100.

12. Fried C. The Roentgen Treatment of Experimental Pneumonia in the Guinea-Pig. Radiology. 1941 08;37(2):197-202. https://doi.org/10.1148/37.2.197

13. Feinendegen LE. Evidence for beneficial low level radiation effects and radiation hormesis. The British Journal of Radiology. 2005 01;78(925):3-7. https://doi.org/10.1259/ bjr $/ 63353075$

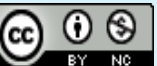

This work is licensed under a Creative Commons AttributionNon Commercial 4.0 International License. 